\title{
SCC Equivalent Resistance: the Relationship for Complementary Buck and Boost and Accurate Calculation for 2-Phase Converters
}

\author{
Yerzhan Mustafa*, Ainur Zhaikhan*, and Alex Ruderman*, Senior Member, IEEE \\ ${ }^{*}$ School of Engineering, Nazarbayev University, Astana, Kazakhstan 010000 \\ (email: yerzhan.mustafa@nu.edu.kz, ainur.zhaikhan@nu.edu.kz, and alexander.ruderman@nu.edu.kz)
}

\begin{abstract}
This paper makes the following contributions to Switched Capacitor Converter (SCC) equivalent (output) resistance analysis. First, it suggests the relationship between equivalent resistances of complementary buck and boost SCC: equivalent resistance of a boost SCC equals that of its buck counterpart times squared boost voltage Target Ratio (Voltage Conversion Ratio). Another contribution is equivalent resistance accurate calculation methodology for an arbitrary 2-phase SCC.
\end{abstract}

Index Terms-DC-DC Power Conversion, Switched Capacitor Circuits, Losses, Equivalent Resistance

\section{INTRODUCTION}

$\mathbf{S}$ WITCHED Capacitor Converter (SCC) is known to have the equivalent circuit for an average output voltage shown in Fig.1,a. The target output voltage is as a product of a DC source voltage and SCC voltage Target Ratio (TR).

An equivalent resistance gives an indication on average converter loss that is an ohmic loss in semiconductor switch (MOSFET) resistances assuming that capacitor losses are negligible ([1], [2]). Capacitor ESR may be easily included if required.

An elegant way to figure out equivalent resistance (at least, in simulation) is to zero DC source and connect $1 \mathrm{~A}$ current source as a load (Fig.1,b). Then a measured average output voltage in Volts represents equivalent resistance in ohms.

For Slow Switching Limit (SSL; hyperbolic dashed line in Fig.2), RC-circuit time constants are much smaller than switching intervals and equivalent resistance is a function of converter switched capacitances and switching frequency only.

For Fast Switching Limit (FSL; horizontal dashed line), RCcircuit time constants are much larger than switching interval durations and equivalent resistance is a function of converter parasitic resistances only.

Suppose there is a buck SCC with $T R<1$ (Fig.3,a). A complementary boost SCC is obtained by swapping the DC source and the load (Fig.3,b). It is well-known that voltage target ratio of a complementary boost SCC is the inverse of its buck counterpart.

This paper suggests that there is a relationship between complementary buck and boost SCC equivalent resistances: equivalent resistance of a boost SCC equals that of its buck counterpart times squared boost voltage TR.

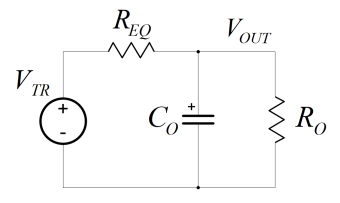

(a) (b)

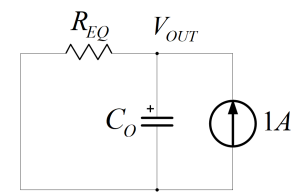

Fig. 1: a) SCC equivalent circuit; b) equivalent resistance measurement in simulation.

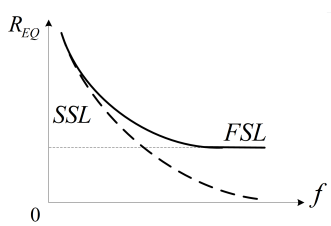

Fig. 2: Equivalent resistance dependence on switching frequency.

Another contribution of the paper is a novel equivalent resistance accurate calculation methodology for an arbitrary 2phase SCC. This methodology is demonstrated for a Fibonacci SCC with 2, 3, and 4 switched capacitors.

\section{The Relationship BETWEen EQuivalent RESISTANCES OF COMPLEMENTARY BUCK AND BoOST SWITCHED CAPACITOR CONVERTERS}

Here is a proposition about the relationship between equivalent resistances of the two SCC: boost SCC equivalent resistance is that of its buck complement times squared TR of the boost SCC (or divided by squared TR of the buck SCC).

This claim neglects the effects of filter capacitor that is valid for relatively high switching frequencies.

A hint may be obtained from a SCC "DC transformer" equivalent circuit (Fig.4) by moving the equivalent resistance across the transformer. However, the authors are unaware of SCC publications explicitly claiming this fact.

For example, the paper by K. Eguchi e.a. [3] presents FSL equivalent resistances for suggested buck SCC with $\mathrm{TR}=1 / 8$; $1 / 13 ; 1 / 15 ; 1 / 16$ and their boost complements (Table II in the paper). By inspection, the proposition of this letter holds but this fact is not stated and discussed though Fig.4 of [3] presents an SCC "DC transformer" equivalent circuit.

A proof must involve calculation of SCC internal losses. The approach of [4], [5] suggests first finding charges flowing through each switched capacitor on switching intervals. For 


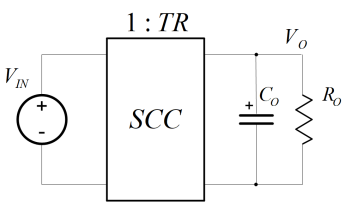

(a)

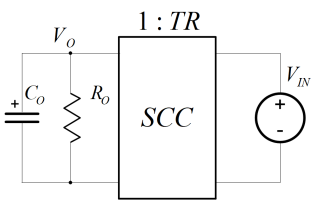

(b)
Fig. 3: Buck SCC (a) and its complementary boost counterpart (b).

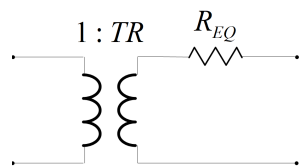

(a)

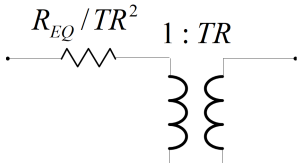

(b)
Fig. 4: DC transformer SCC equivalent circuit: a) buck; b) boost.

that, a set of linear equations must be generated based on: 1) steady-state conditions - a net charge of a switched capacitor must be zero on a switching period; 2) Kirchhoff current law for series-parallel switched capacitor connections.

The last equation to be added is that of a total charge (current) into the load on a switching period. While the first charge balance - equations set the proportions between the charges of switched capacitors, the last one defines their absolute values (scale) based on the net charge (current) supplied to the load.

Let's assume ideal DC source (decoupling capacitance) and pure DC output voltage that is a common assumption for analysis of DC-DC converters; reasonably small output voltage ripple can be analyzed later on. Then for each switching phase SCC may be accurately represented by a (high order) RCcircuit. Once all the charges through switched capacitors are determined by solving linear algebraic equations, they can be spread over switching intervals using exponential solutions of respective RC-circuit differential equations.

Overall loss due to exponential currents in the circuit resistances is calculated by taking squared current integrals and equivalent resistance is found by dividing overall loss by squared output current (that is effectively cancelled).

The logic of the suggested proof is as follows:

- switched capacitor charge balance equations for two complementary SCC are identical for the same phases;

- the difference is in the last equations that set the charges absolute values based on the net charge to the load;

- for the same load current, individual charges of boost SCC are those of buck SCC times boost converter TR (can be verified by inspection);

- as the equivalent RC-circuits are the same for both SCC, buck capacitor currents are scaled with the boost SCC TR; it means that overall loss is scaled with squared boost TR for the same load DC current that proves the main proposition.

This basic idea is illustrated below by examples using an elementary 2 capacitor Fibonacci SCC (Fig.5).

\section{Fibonacci SCC with Multiphase Switching}

Consider Fibonacci buck SCC multiphase switching ([6]) to produce $\mathrm{TR}=1 / 3$. Available switching topologies (phases) are

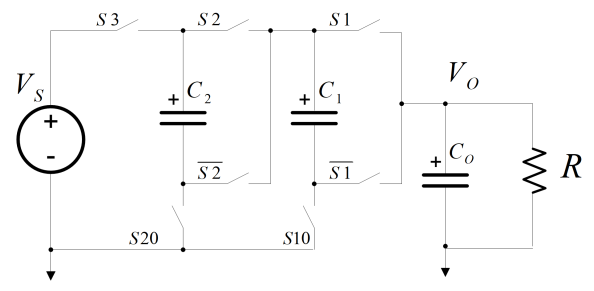

Fig. 5: Fibonacci buck SCC with 2 capacitors.

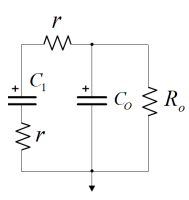

(a)

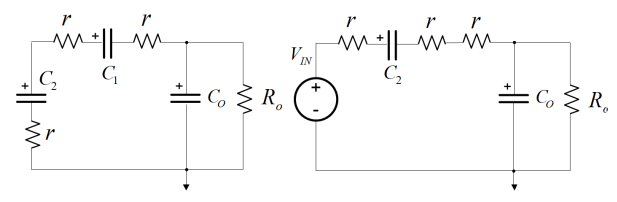

(b) (c)
Fig. 6: Multiphase Fibonacci buck SCC topologies. shown in Fig.6.

Capacitor charge balance equations for the three phase charges to the load

$q_{1}-q_{2}=0 ; \quad q_{2}-q_{3}=0 ; \quad q_{1}+q_{2}+q_{3}=Q$.

The first two equations are about charge balance of individual switched capacitors while the last one - about the net charge to the load during the switching period $T, Q / T=I$ load DC current. The solution of (1) is given by

$q_{1}=Q / 3 ; \quad q_{2}=Q / 3 ; \quad q_{3}=Q / 3$.

that means balanced multiphase switching ([6], [7]).

Three equal charges (2) are spread over switching intervals with their respective time constants ([4], [5]) according to equivalent first order RC-circuits (Fig. 6; output voltage is assumed pure DC; switched capacitances $C_{1}=C_{2}=C$ )

$T_{1}=2 r C ; \quad T_{2}=(3 / 2) r C ; \quad T_{3}=3 r C$.

By the methodology [5], [6], the equivalent resistance expression becomes

$R_{E Q}=\frac{1}{18 f C}\left[\operatorname{coth}\left(\frac{\beta}{4}\right)+2 \operatorname{coth}\left(\frac{\beta}{3}\right)+\operatorname{coth}\left(\frac{\beta}{6}\right)\right]$,

where $\beta=1 /(3 r C f), f$ - switching frequency.

For the complementary boost SCC, capacitor charge balance equations differ from (1) in the last one because the load is connected only during the third stage (Fig.6) -

$q_{1}-q_{2}=0 ; \quad q_{2}-q_{3}=0 ; \quad q_{3}=Q$.

The solution of (5) is (2) multiplied by the boost $\mathrm{TR}=3$ -

$q_{1}=Q ; \quad q_{2}=Q ; \quad q_{3}=Q$.

This way, complementary boost SCC equivalent resistance is (4) multiplied by 9 . Equivalent resistance measurement results according to Fig.1,b are presented in Fig.7 for $C=10 \mu F ; C o=100 \mu F ; r=1 \Omega ; f=10 \mathrm{kHz}$. The measured equivalent resistances amount to 3.28 (practically the same as calculated by (4)) and $29.6 \Omega$ respectively with the ratio being very close to predicted theoretical value of 9 .

The next Section presents accurate equivalent resistance calculation methodology for a 2-phase SCC demonstrated for a Fibonacci SCC with 2, 3, and 4 switched capacitors. 


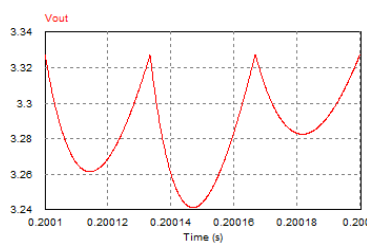

(a)

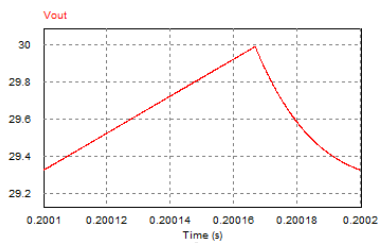

(b)
Fig. 7: Multiphase SCC equivalent resistance measurements: a) buck; b) boost.

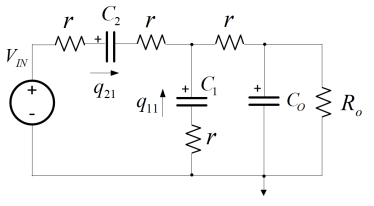

(a)

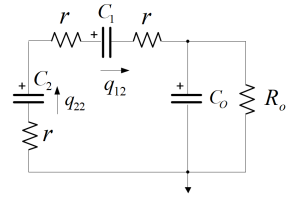

(b)
Fig. 8: 2-phase Fibonacci SCC topologies: a) phase 1; b) phase 2.

\section{FibOnACCI SCC With 2-Phase Switching}

\section{A. 2-Phase Fibonacci SCC with 2 Switched Capacitors}

Classic 2-phase Fibonacci buck SCC generates TR=1/3 with phase topologies shown in Fig.8.

Capacitor charge balance equations are written as (Fig.8)

$q_{11}-q_{12}=0 ; \quad q_{21}-q_{22}=0 ; \quad q_{12}-q_{22}=0 ;$

$q_{11}+q_{21}+q_{12}=Q$,

with the solution being

$q_{11}=q_{12}=q_{21}=q_{22}=Q / 3$.

During phase 1 (Fig.8,a) the charge delivered to the load is $2 Q / 3$, during phase 2 (Fig.8,b) - $Q / 3$.

For the complementary boost SCC, the last charge balance equation in (7) is transformed into $q_{21}=Q$ and the solution -

$q_{11}=q_{12}=q_{21}=q_{22}=Q$.

Assuming pure DC output voltage, phase 1 (Fig.8,a) equivalent 2 nd order circuit for natural response calculation becomes as in Fig.9 with two time constants for equal capacitances being

$T_{1}=1.382 r C ; \quad T_{2}=3.618 r C$.

Let's seek phase 1 capacitor currents as

$i_{11}(t)=a_{11} \exp \left(-t / T_{1}\right)+a_{12} \exp \left(-t / T_{2}\right) ;$

$i_{21}(t)=a_{21} \exp \left(-t / T_{1}\right)+a_{22} \exp \left(-t / T_{2}\right)$.

To find four unknown coefficients in (11), one needs a set of four linear equations. The first one is based on the capacitor charges equality $q_{11}=q_{12}(8)$ -

$\int_{0}^{T / 2} i_{11}(t) d t=\int_{0}^{T / 2} i_{21}(t) d t$

$T$ - switching period.

Two more equations come from the partial Kirchhoff voltage law for the left mesh in Fig.8, a for two exponential terms (DC terms balance is provided by capacitor initial voltages) -

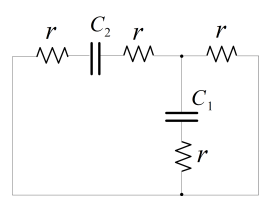

Fig. 9: Phase 1 equivalent circuit.

$i_{11} r+\frac{1}{C_{1}} \int_{0}^{t} i_{11}(t) d t=i_{21}(2 r)+\frac{1}{C_{2}} \int_{0}^{t} i_{21}(t) d t$.

Partial Kirchhoff voltage law for the right mesh in Fig.8,a will be fulfilled automatically.

The last - scaling - equation comes from (8) -

$\int_{0}^{T / 2} i_{11}(t) d t=Q / 3$.

Once the coefficients of (11) proportional to $Q$ are found, power loss in all resistances for 2 phases can be calculated by taking squared current integrals. Phase 2 losses are addressed similar to multiphase (series capacitances connection).

Finally, the equivalent resistance expression is found as

$R_{E Q}=\frac{1}{170 f C} \operatorname{coth}\left(\frac{1}{5.53 r C f}\right)+\frac{1}{9.50 f C} \operatorname{coth}\left(\frac{1}{14.5 r C f}\right)+$ $+\frac{1}{9 f C} \operatorname{coth}\left(\frac{1}{6 r C f}\right)$.

The first two terms in (15) are due to squared exponential terms of phase 1 currents (11). The first term is due to the smaller time constant in (9) and its contribution is less.

One can expect another term due to the cross-product when squaring currents (11). However, these terms cancel when calculating overall phase 1 power loss.

The measured equivalent resistances (Fig.10) amount to 3.00 (3.01 calculated by (15)) and $26.9 \Omega$ with the ratio being very close to 9 as predicted by the theory.

Phase 1 simulated capacitor currents (Fig.11) are accurately reproduced by two exponent model (11), the same for phase 2 - by a single exponential term.

\section{B. 2-Phase Fibonacci SCC with 3 Switched Capacitors}

A 2-phase 3 switched capacitor Fibonacci SCC (Fig.12) generates $\mathrm{TR}=1 / 5$. Phase topologies are shown in Fig.13.

Charge flow equations become

$$
\begin{aligned}
& q_{11}-q_{12}=0 ; \quad q_{21}-q_{22}=0 ; \quad q_{31}-q_{32}=0 \\
& q_{31}+q_{21}-q_{11}=0 ; \quad q_{32}-q_{22}=0 \\
& q_{11}+q_{12}+q_{22}=Q
\end{aligned}
$$

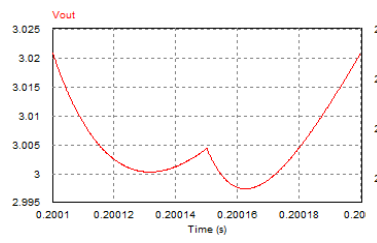

(a)

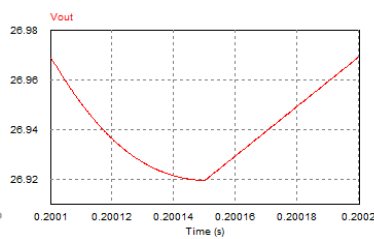

(b)
Fig. 10: 2-phase SCC with 2 capacitors equivalent resistance measurements: a) buck; b) boost. 


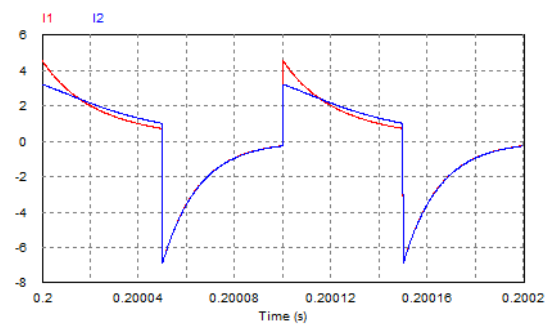

Fig. 11: 2-phase SCC capacitor currents.

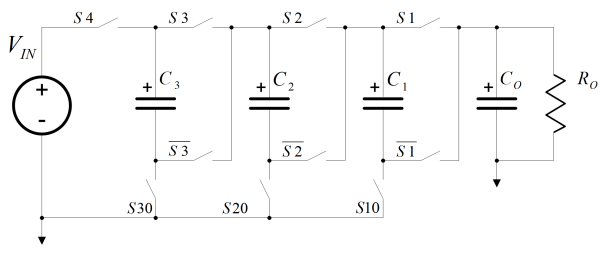

Fig. 12: Fibonacci SCC with 3 switched capacitors.

with the solution being

$q_{11}=q_{12}=2 Q / 5$

$q_{21}=q_{22}=q_{31}=q_{32}=Q / 5$.

During Phase 1 the charge delivered to the load is $2 Q / 5$, during Phase $2-3 Q / 5$.

Assuming pure DC output voltage, Phase 1 and 2 equivalent RC-circuits become as shown in Fig.14.

Though Phase 1 circuit (Fig.14,a) is the 3rd order one, there are only 2 time constants because 3 capacitors are connected to the same node.

Assuming equal switched capacitances, Phase 1 and 2 time constants are found as

$T_{11}=1.33 r C ; \quad T_{12}=2.0 r C ;$

$T_{21}=1.293 r C ; \quad T_{22}=2.707 r C$.

Phase 1 and 2 capacitor currents are sought in the form

$i_{21}(t)=a_{211} \exp \left(-t / T_{11}\right)+a_{212} \exp \left(-t / T_{12}\right) ;$

$i_{31}(t)=a_{311} \exp \left(-t / T_{11}\right)+a_{312} \exp \left(-t / T_{12}\right) ;$

$i_{11}(t)=i_{21}(t)+i_{31}(t)$,

and

$i_{12}(t)=a_{121} \exp \left(-t / T_{21}\right)+a_{122} \exp \left(-t / T_{22}\right) ;$

$i_{22}(t)=a_{221} \exp \left(-t / T_{21}\right)+a_{222} \exp \left(-t / T_{22}\right) ;$

$i_{32}(t)=i_{22}(t)$.

To find unknown coefficients in (19), (20), sets of linear equations are generated based on:

- capacitor charges equalities (17) - calculating time integrals of (19), (20) on half a period and equating the same exponential term coefficients;

- partial Kirchhoff voltage laws for the left meshes (Fig.13, $a$ and $b$ ) for 2 exponential terms (partial Kirchhoff voltage law for unused right meshes will be fulfilled automatically that can be checked by direct inspection);

- scaling equation from (17) setting capacitor current time integral on half a period equal to $2 Q / 5$ or $Q / 5$.

Once the coefficients of (19), (20) are found from the linear set of equations, power loss in all resistances for 2 phases has to be calculated by taking squared current integrals

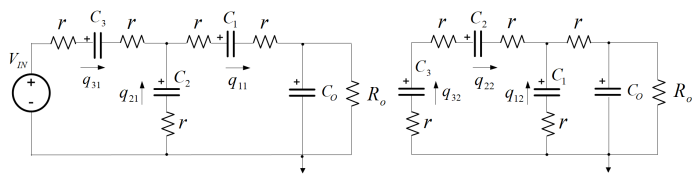

(a)

(b)

Fig. 13: 2-phase Fibonacci SCC topologies: a - phase 1; b phase 2.

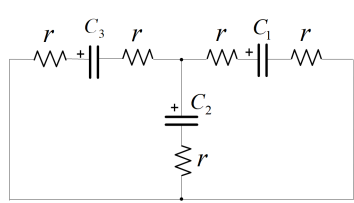

(a)

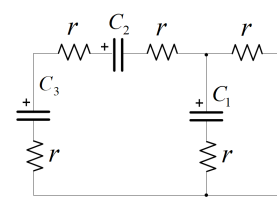

(b)
Fig. 14: Phase 1 (a) and 2 (b) equivalent circuits for time constants calculation.

to proceed to equivalent resistance. Finally, the equivalent resistance expression is determined as

$R_{E Q}=\frac{1}{33.3 f C} \operatorname{coth}\left(\frac{1}{5.53 r C f}\right)+\frac{1}{11.1 f C} \operatorname{coth}\left(\frac{1}{8 r C f}\right)+$

$+\frac{1}{8.58 f C} \operatorname{coth}\left(\frac{1}{10.8 r C f}\right)+\frac{1}{291 f C} \operatorname{coth}\left(\frac{1}{5.17 r C f}\right)$.

The first two terms of (21) represent the contribution of 2 squared exponential terms of Phase 1 currents, the last two that of Phase 2.

Exponential cross-product terms due to squaring currents (19), (20) cancel when calculating overall power losses.

Measured average output voltage that represents equivalent resistance in ohms for parameters $C=10 \mu F ; C o=100 \mu F$; $r=1 \Omega ; f=10 \mathrm{kHz}$ is presented in Fig. 15 .

The measured equivalent resistance amounts to $3.00 \Omega(3.01$ calculated by formula (21)).

Simulated SCC output voltage, current to the filtered load, and switched capacitor currents are shown in Fig. 16 for source voltage of $5 \mathrm{~V}$ and active load equal to equivalent resistance.

Switched capacitor currents (Fig.16,b) are perfectly reconstructed using 2nd order RC-circuit models (19),(20).

\section{2-Phase Fibonacci SCC with 4 Switched Capacitors}

A 2-phase 4 switched capacitor Fibonacci SCC (Fig.17) generates TR=1/8. Phase topologies are shown in Fig.18.

Capacitor charge balance equations become

$$
\begin{aligned}
& q_{11}-q_{12}=0 ; \quad q_{21}-q_{22}=0 ; \quad q_{31}-q_{32}=0 \\
& q_{41}-q_{42}=0 ; \quad q_{41}+q_{31}-q_{21}=0 ; \\
& q_{42}-q_{32}=0 ; \quad q_{32}+q_{22}-q_{12}=0 ; \\
& q_{11}+q_{21}+q_{12}=Q
\end{aligned}
$$

with the solution being

$$
\begin{aligned}
& q_{11}=q_{12}=3 Q / 8 \\
& q_{21}=q_{22}=2 Q / 8 \\
& q_{31}=q_{32}=q_{41}=q_{42}=Q / 8
\end{aligned}
$$

During Phase 1 the charge delivered to the load is $5 Q / 8$, during Phase $2-3 Q / 8$. 


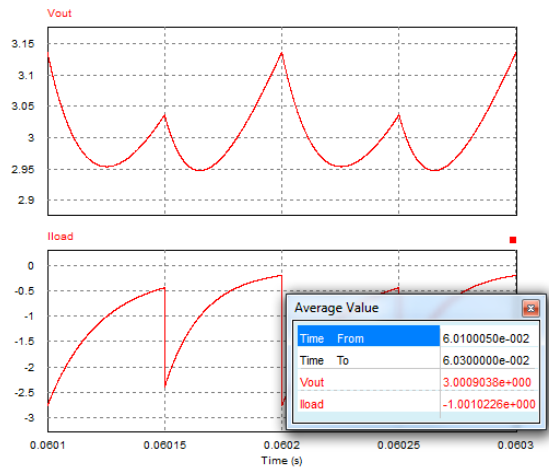

Fig. 15: Equivalent resistance measurement for 2-phase Fibonacci SCC with 3 capacitors.

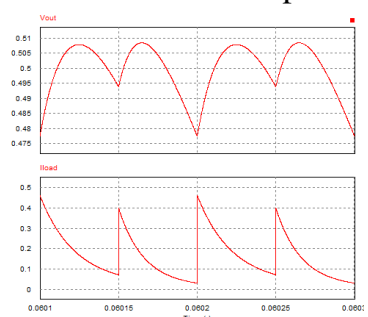

(a)

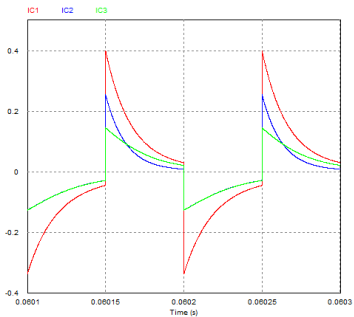

(b)
Fig. 16: 2-phase 3 capacitor SCC simulation results: a) output voltage and current to the filtered load; b) switched capacitor currents.

Assuming pure DC output voltage, Phase 1 and 2 equivalent RC-circuits become as shown in Fig. 18.

Though Phase 1 circuit (Fig.18,a) is the 4th order one, there are only 3 time constants because 3 capacitors are connected to the same node. For Phase 2 circuit (Fig.18,b), there are 2 time constants for the same reason.

Assuming equal switched capacitances, Phase 1 and 2 time constants are found as

$T_{11}=1.258 r C ; \quad T_{12}=1.605 r C ; \quad T_{13}=3.137 r C ;$

$T_{21}=1.724 r C ; \quad T_{22}=1.276 r C$.

Phase 1 and 2 capacitor currents are sought in the form

$i_{11}(t)=a_{111} \exp \left(-t / T_{11}\right)+a_{112} \exp \left(-t / T_{12}\right)+$

$+a_{113} \exp \left(-t / T_{13}\right)$

$i_{31}(t)=a_{311} \exp \left(-t / T_{11}\right)+a_{312} \exp \left(-t / T_{12}\right)+$

$+a_{313} \exp \left(-t / T_{13}\right)$

$i_{41}(t)=a_{411} \exp \left(-t / T_{11}\right)+a_{412} \exp \left(-t / T_{12}\right)+$

$+a_{413} \exp \left(-t / T_{13}\right)$

$i_{21}(t)=i_{31}(t)+i_{41}(t)$,

and

$i_{22}(t)=a_{221} \exp \left(-t / T_{21}\right)+a_{222} \exp \left(-t / T_{22}\right) ;$

$i_{32}(t)=a_{321} \exp \left(-t / T_{21}\right)+a_{322} \exp \left(-t / T_{22}\right) ;$

$i_{32}(t)=i_{42}(t)$;

$i_{12}(t)=i_{22}(t)+i_{32}(t)$.

To find unknown coefficients in (25), (26), sets of linear equations are generated based on:

- capacitor charges equalities (23) - calculating time integrals of (25), (26) on half a period and equating the same

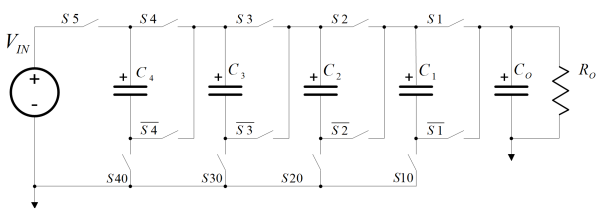

Fig. 17: Fibonacci SCC with 4 switched capacitors.

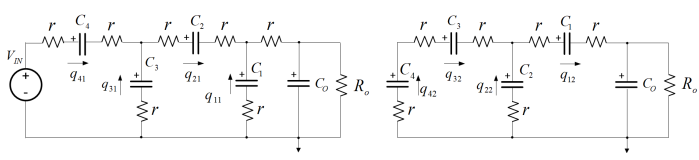

(a)

(b)

Fig. 18: 2-phase Fibonacci SCC topologies: a - phase 1; b phase 2.

exponential term coefficients;

- partial Kirchhoff voltage laws for 2 left meshes (Fig.18, a) and the left mesh (Fig.18, b) for 3 exponential terms of Phase 1 and 2 terms of Phase 2;

- scaling equation from (23) setting capacitor current time integral on half a period equal to $3 Q / 8$ or $2 Q / 8$ or $Q / 8$.

Once the coefficients of (25), (26) are found from the linear set of equations, power loss in all resistances for 2 phases has to be calculated by taking squared current integrals to proceed to equivalent resistance. Finally, the equivalent resistance expression is determined as

$R_{E Q}=\frac{1}{642 f C} \operatorname{coth}\left(\frac{1}{5.03 r C f}\right)+\frac{1}{116.5 f C} \operatorname{coth}\left(\frac{1}{6.42 r C f}\right)+$ $+\frac{1}{9.34 f C} \operatorname{coth}\left(\frac{1}{12.5 r C f}\right)+\frac{1}{67.0 f C} \operatorname{coth}\left(\frac{1}{5.11 r C f}\right)+$

$+\frac{1}{9.78 f C} \operatorname{coth}\left(\frac{1}{6.89 r C f}\right)$.

The first three terms of (27) represent the contribution of 3 squared exponential terms of Phase 1 currents, the last two 2 squared terms of Phase 2.

Exponential cross-product terms due to squaring currents (25), (26) cancel when calculating overall power losses.

Measured average output voltage that represents equivalent resistance in ohms for parameters $C=10 \mu \mathrm{F} ; C o=100 \mu \mathrm{F}$; $r=1 \Omega ; f=20 \mathrm{kHz}$ is presented in Fig. 20 .

The measured equivalent resistance amounts to $2.41 \Omega(2.42$ calculated by formula (27)).

Simulated SCC output voltage, current to the filtered load, and switched capacitor currents are shown in Fig.21 for source voltage of $8 \mathrm{~V}$ and active load equal to equivalent resistance.

Switched capacitor currents (Fig.21,b) are perfectly reconstructed using 3rd and 2nd order RC-circuit models (25),(26).

\section{COMparison of ACCURATE AND Approximate EQUIVALENT RESISTANCES}

The majority of researchers is convinced that accurate SCC equivalent resistance calculation (even under infinite filter

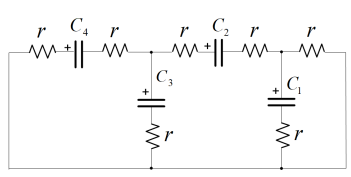

(a)

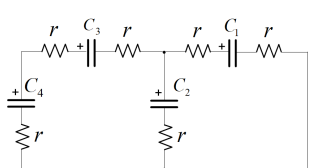

(b)
Fig. 19: Phase 1 (a) and 2 (b) equivalent circuits for time 


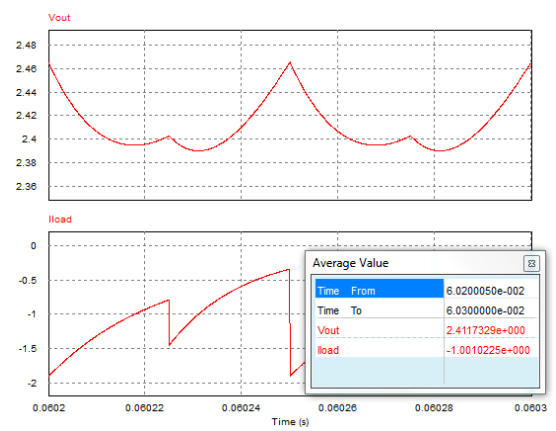

Fig. 20: Equivalent resistance measurement for 2-phase Fibonacci SCC with 4 capacitors.

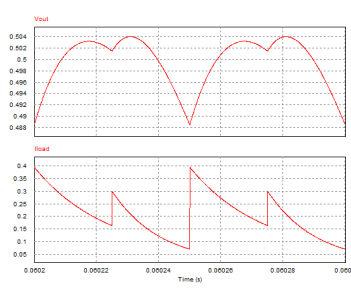

(a)

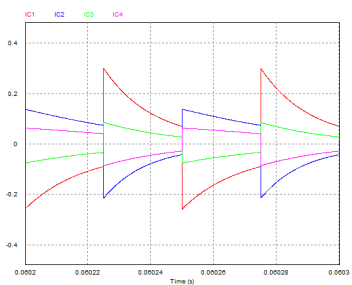

(b)
Fig. 21: 2-phase 4 capacitor SCC simulation results: a) output voltage and current to the filtered load; b) switched capacitor currents.

capacitance assumption) is a difficult task [8]. Most popular Seeman-Maksimovic approximate formula is [8]

$R_{E Q}=\left[\left(R_{S S L}\right)^{2}+\left(R_{F S L}\right)^{2}\right]^{1 / 2}$.

where $R_{S S L}$ - SSL equivalent resistance approximation; $R_{F S L}$ - FSL one.

Recently, M. Makowski suggested another formula [9] -

$R_{E Q}=\left[\left(R_{S S L}\right)^{p}+\left(R_{F S L}\right)^{p}\right]^{1 / p} ; \quad p=2.545$.

The results of accurate equivalent resistance calculation from the previous sections (15), (21), (27) are compared with their approximations (28), (29) in Fig.22-24.

\section{CONCLUSION}

This paper suggests that equivalent resistance of a boost SCC equals that of its buck complementary counterpart times squared boost voltage Target Ratio. The proposition is solidified by theoretical calculations and simulation examples for a 2 capacitor Fibonacci SCC with multiphase switching and 2, 3 and 4 capacitor Fibonacci SCC with 2-phase switching.

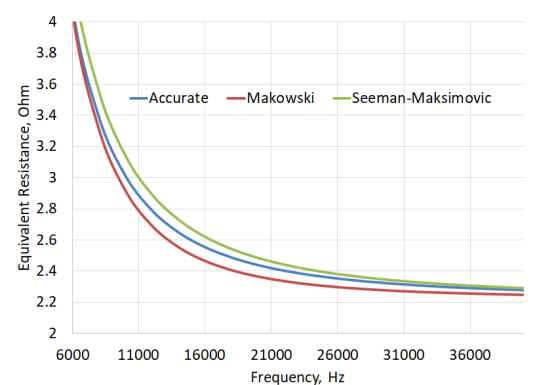

Fig. 22: Comparison of accurate and approximate equivalent

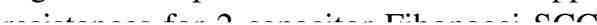

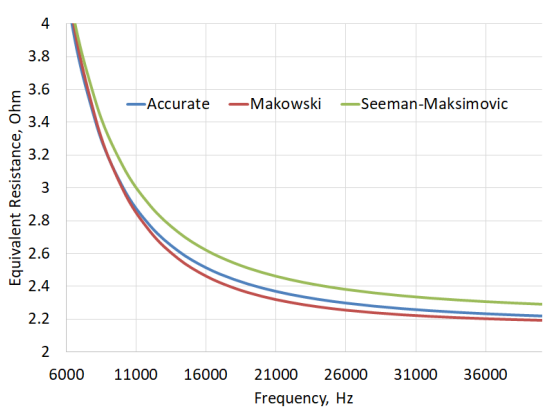

Fig. 23: Comparison of accurate and approximate equivalent resistances for 3 capacitor Fibonacci SCC

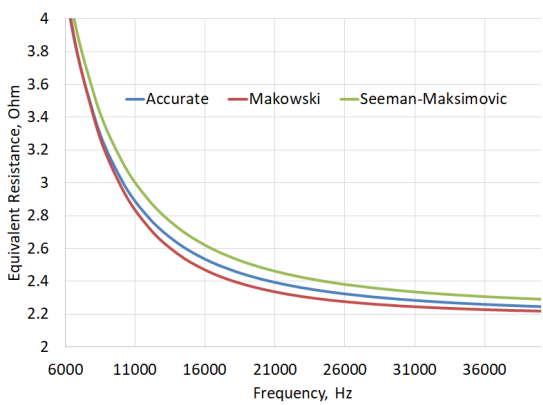

Fig. 24: Comparison of accurate and approximate equivalent resistances for 4 capacitor Fibonacci SCC

The proposed accurate equivalent resistance calculation methodology for a 2-phase SCC is demonstrated for Fibonacci SCC with 2, 3, and 4 switched capacitors. Accurate equivalent resistance is compared with Seeman-Maksimovic and Makowski approximations in a broad frequency range.

\section{REFERENCES}

[1] S. Ben-Yaakov, "On the influence of switch resistances on switched capacitor converters losses," IEEE Trans. Ind. Electron., vol. 59, no. 1, Jan. 2012, pp. 638-840.

[2] S. Ben-Yaakov and M. Evzelman, "Average-current-based conduction losses model of switched capacitor converters," IEEE Trans. Power Electronics, vol. 28, no. 7, July 2013, pp. 3341-3352.

[3] K. Eguchi, S. Hirata, M. Shimoji, and H. Zhu, "Design of a Step-Up/Step-Down (=2,3,)-Fibonacci DC-DC Converter Designed by Switched-Capacitor Techniques," in Proc. 5th Int. Conf. on Intelligent Networks and Intelligent Systems, Jan. 2014, pp. 170-173.

[4] A. Kushnerov, "High-efficiency self-adjusting switched capacitor DC-DC converter with binary resolution," MSc Thesis, Ben-Gurion University of the Negev, 2009, 115 p.

[5] A. Kushnerov, "Multiphase Fibonacci switched capacitor converters," IEEE J. Emerg. Sel. Topics Power Electron., vol. 2, no. 3, Sep. 2014, pp. $460-465$.

[6] A. Junussov and A. Ruderman, "Analysis of a reconfigurable Fibonacci switched capacitor converter with a multiphase balanced switching," in Proc. Int. Conf. Power Eng. Energy Elec. Drives (POWERENG), May 2015, pp. 164-169.

[7] A. Zhaikhan, A. Daulbayeva, A. Berdygozhin, and A. Ruderman, "Novel voltage target ratios and minimal norm principle for reconfigurable multiphase single- and dual-output switched capacitor converters", in Proc. IET Int. Conf. on Power Electron., Machines and Drives (PEMD), Apr. 2016, pp. $1-8$.

[8] M. D. Seeman, "A design methodology for switched-capacitor DCDC converters," Ph.D. dissertation, EECS Dep., Univ. California, Berkeley, Tech. Rep. UCB/EECS-2009-78, May 2009.

[9] M. S. Makowski, "A note on resistive models of switched-capacitor DC-DC converters: Unified incremental-graph-based formulas given," in International Conference on Signals and Electronic Systems (ICSES), Sep. 2012, pp. 1-4. 